\title{
Hipertensión pulmonar e hipoxemia grave en recién nacidos
}

\author{
FERNANDO SILVERA ${ }^{1,2,3}$, ALICIA MELE ${ }^{1,2}$, MARIELA COSTAS ${ }^{1,2}$, \\ MARIELA VIÑA ${ }^{1,2}$, MAGDALENA HERMIDA ${ }^{2}$, ENRIQUE DI LUCCI ${ }^{1,2}$, \\ RUTH KESHISHIAN ${ }^{1,2}$, EDUARDO MAYANS ${ }^{1,2}$, GONZALO GIAMBRUNO ${ }^{4}$ \\ 1. Ex asistente de Neonatología. \\ 2. Neonatólogo. \\ 3. Asistente Area Básica del departamento de Neonatología del Hospital de Clínicas. Facultad de Medicina. \\ 4. Prof. Adjunto de Neonatología. Supervisor neonatal de AESPM. Asociación Española Primera de Socorros Mutuos \\ (AESPM).
}

\section{ABSTRACT \\ Severe hypoxemia and pulmonary hypertension in newborns}

Inhaled nitric oxide (iNO) through direct pulmonary vasodilator action and without sistemyc effects, is the gold standard treatment for pulmonary hypertension of the newborn (HTPP). This work was designed with the aim of evaluating the response to this treatment in 16 newborn patients in an intensive care unit, by changes in oxygen saturation (SO2), medium airway pressure (MAP) and inspirited fraction of O2 (FiO2). Two groups were anaylized: 1) under iNO treatment during the first 72 hs of life, where the most frequent diagnosis was meconium aspiration syndrome and 2) newborns with severe respiratory failure $(\mathrm{n}=3)$, refractary to treatment, in which iNO was begun alter 14 days of life. Among patients of group 1 it was evident an increase in SO2 after the first 6 hs of treatment with iNO. The decrease FiO2 and MAP occurred later. The mortality rate was $23 \%(2 / 13)$ in this group. In group 2 the SO2 increased, leading to a decrease in ventilatory support in the first 72 hours of treatment in one case. iNO doses were lesser than 20 ppm in $8 / 13$ patients, there were neither platelet impairment, nor bleeding or metahemoglobinemia. There was no rebound effect after finishing iNO therapy from minimum doses $(2 \mathrm{ppm})$. In this unit the inclusion of iNO treatment in HTPP or respiratory failure was associated with improvement in $\mathrm{SO} 2$ and a decrease in ventilatory support, without adverse effects resulting in a benefit over the globally support therapy.

(Key words: Hypertension, pulmonary, nitric oxide, anoxemia, infant, newborn).

Arch Pediatr Urug 2007; 78(4): 270-280

\section{Correspondencia a:}

Viviana Romanin

E-mail:vromanin@arnet.com.ar

La idea original y la confección del Protocolo, estuvieron a cargo de la Organización Panamericana de la Salud.

El Protocolo fue aprobado por los Comités de Docencia e Investigación y Bioética, de los hospitales de Niños "Dr. Ricardo Gutiérrez" y "Dr. Pedro de Elizalde". 


\section{RESUMEN}

El óxido nítrico inhalado $(\mathrm{ONi})$, mediante vasodilatación pulmonar directa y carencia de efectos sistémicos, constituye el tratamiento ideal para la hipertensión pulmonar del recién nacido (HTPP). Para evaluar la respuesta a dicho tratamiento se presenta la evolución de 16 pacientes internados en una unidad de cuidados intensivos neonatales valorada por cambios en la saturación de $\mathrm{O} 2$ ( $\mathrm{SO} 2)$, presión media en la vía aérea (PMVA) y fracción inspirada de O2 (Fio2). Se analizaron dos grupos: 1) bajo tratamiento con ONi en las primeras 72 horas de vida $(\mathrm{n}=13)$-su diagnóstico más frecuente fue aspiración de líquido meconial (SALAM)-; y 2) recién nacidos con insuficiencia respiratoria grave ( $\mathrm{n}=3)$, refractaria al tratamiento, en los que se inició ONi luego de 14 días de vida. En el grupo 1 se constató un aumento significativo de la SO2 a partir de las primeras 6 horas del tratamiento con ONi. El descenso de Fio2 y de PMVA fue más tardío, con una mortalidad de 23\% (2/13). En el grupo 2 se elevó la SO2, permitiendo disminuir el soporte ventilatorio en las primeras 72 horas de la terapia en un caso. Las dosis de ONi utilizadas fueron menores a las $20 \mathrm{ppm}$ en $8 / 13$ pacientes, no se observaron alteraciones plaquetarias, sangrados, metahemoglobinemia ni se observó efecto rebote al suspender el ONi desde dosis mínimas ( $2 \mathrm{ppm})$. En esta unidad, la inclusión de ONi en el tratamiento de la HTPP o insuficiencia respiratoria se asoció con mejoría de la SO2 y a descenso del soporte ventilatorio, sin efectos adversos, con beneficios sobre la terapia global instituida.

(Palabras clave: Hipertensión pulmonar, óxido nítrico, anoxemia, recién nacido).

Arch Pediatr Urug 2007; 78(4): 270-280

ESTE TRABAJO LO PUEDE ENCONTRAR EN EXTENSO EN WWW.SCIELO.CL 